\title{
Adición de Enterococcus faecium mejora poblaciones celulares inmunes y anticuerpos vacunales de lechones destetos*
}

\author{
Víctor H. Herrera Franco**, Johana Ciro Galeano**, Jaime Parra Suescún***
}

\section{Resumen}

Introducción. Durante el destete los animales son sometidos a diferentes tipos de estrés en el momento que el lechón no es capaz de producir una adecuada actividad inmunológica, debido a que la inmunidad pasiva adquirida del calostro va disminuyendo y el lechón presenta una baja respuesta inmunológica durante el período posdestete. Como una alternativa al uso de antibióticos contra las bacterias que provocan alteraciones del sistema inmune y sin afectar la salud animal y humana, se ha propuesto la utilización de microorganismos vivos conocidos como probióticos. Objetivo. Determinar el efecto de probióticos (Lactobacillus acidophilus, Lactobacillus casei y Enterococcus faecium) sobre las poblaciones de células inmunes a nivel sanguíneo de cerdos destetos. Materiales y métodos. Se experimentó con 80 lechones destetados a los 21 días de vida, alimentados con dos dietas: dieta comercial con y sin la adición de antibiótico; a esta última se adicionaron los diferentes probióticos en el agua de bebida. Se tomó muestra sanguínea de la vena yugular los días 1, 15, y 30 posdestete. Se utilizó un diseño por bloques al azar en un arreglo de medidas repetidas en el tiempo. Cada animal fue aleatorizado a uno de 15 tratamientos (5 dietas* 3 tiempos). Resultados. Aumento significativo $(P<0.01)$ en el número de células inmunes sanguíneas y del número de anticuerpos vacunales en los animales que consumieron $E$. faecium, frente a aquellos que consumieron la dieta con antibiótico. Conclusiones. El probiótico E. faecium mejora la respuesta del animal frente a estímulos estresantes como el destete, al mostrar un aumento controlado de células inmunes sanguíneas.
Palabras clave: células inmunes, destete, lechones, probióticos.

\author{
The addition of enterococcus faecium \\ improves immune cellular populations and \\ vaccine antibodies in weaners
}

\begin{abstract}
Introduction. Animals are subjected, at the moment the sucking pig cannot produce an adequate immunological activity, to several stress types during waning, due to the fact that the passive immunity acquired at the colostrum is gradually reduced and the sucking pig has a low immunological response during post weaning. Objective. Determine the effect of probiotics (Lactobacillus acidophilus, Lactobacillus casei and Enterococcus faecium) on the populations of immune cells in the blood of weaners. Materials and methods. The experiment was made with 80 weaners with 2 days of age, fed with two diets: commercial diet with and without probiotics. For the first, different probiotics were added in the weaner's water. A blood sample was taken from the jugular vein at the 1,15 and 30 post weaning days. A randomized blocks design was used, arranged with repeated measures during time. Every animal was randomized to one of 15 treatments (5 diets*3times). Results. A significant increase $(P<0.01)$ in the number of immune blood cells and of the number of vaccine antibodies in the animals that consumed E. faecium, in comparison to those that consumed the diet with antibiotics. Conclusions. E. faecium improves the animal's
\end{abstract}

\footnotetext{
* $\quad$ Artículo derivado del proyecto de investigación titulado "Efecto de la adición de diferentes cepas probióticas tipo gras (L. acidophilus, L. case y E. faecium) sobre la población bacteriana intestinal, y su relación con variables inmunológicas en lechones recién destetados", realizada entre 2014-2015 y financiado por la Universidad Nacional de Colombia, Sede Medellín (DIME). Código QUIPU 201010013322.

** Profesor Auxiliar. Facultad de Medicina Veterinaria y Zootecnia. Universidad Autónoma de Las Américas.

*** Profesor Asociado. Departamento de Producción Animal, Facultad de Ciencias Agrarias, Universidad Nacional de Colombia, Sede Medellín. Grupo Biodiversidad y Genética Molecular BIOGEM.
}

Autor para correspondencia: Víctor H. Herrera Franco, e-mail: vhherrer@unal.edu.co Artículo recibido: 09/02/2016; Artículo aprobado: 15/11/2016 
response to stressful stimuli such as weaning, having a controlled increase of immune blood cells.

Key words: immune cells, weaning, weaners, probiotics.

\section{Adição de enterococcus faecium melhora populações celulares imunes e anticorpos vacunais de leitões desmamados}

\section{Resumo}

Introdução. Durante o desmame os animais são submetidos a diferentes tipos de estresse no momento que o leitão não é capaz de produzir sua atividade imunológica adequada, devido a que a imunidade passiva adquirida do colostro vá diminuindo e o leitão apresenta uma baixa resposta imunológica durante o período pós-desmame. Como uma alternativa ao uso de antibióticos contra as bactérias que provocam alterações do sistema imune e sem afetar a saúde animal e humana, se há proposto a utilização de microrganismos vivos conhecidos como probióticos. Objetivo. Determinar o efeito de probióticos (Lactobacillus acidophilus, Lactobacillus casei e Enterococcus faecium) sobre as populações de células imunes a nível sanguíneo de porcos desmamados. Materiais e métodos. Se experimentou com 80 leitões desmamados aos 21 dias de vida, alimentados com duas dietas: dieta comercial com e sem a adição de antibiótico; a esta última se adicionaram os diferentes probióticos na água para beber. Se tomou amostra sanguínea da veia jugular nos dias 1,15 , e 30 pós-desmame. Se utilizou um desenho por blocos ao azar num acordo de medidas repetidas no tempo. Cada animal foi aleatorizado a um de 15 tratamentos (5dietas*3tempos). Resultados. Aumento significativo $(P<0.01)$ no número de células imunes sanguíneas e do número de anticorpos vacunais nos animais que consumiram $E$. faecium, frente a aqueles que consumiram a dieta com antibiótico. Conclusões. $O$ probiótico $E$. faecium melhora a resposta do animal frente a estímulos estressantes como o desmame, ao mostrar um aumento controlado de células imunes sanguíneas.

Palavras chave: células imunes, desmame, leitões, probióticos.

\section{Introducción}

El lechón recién nacido depende de la inmunidad pasiva suministrada por la madre. Al nacer, el animal recibe inmunoglobulinas (Ig), que son ingredientes inmunizantes del calostro de vital importancia y determinantes para la defensa y prevención frente a infecciones que pueden atacar al lechón, y que atraviesan la pared intestinal durante las primeras horas de vida (apertura intestinal) y proporcionan inmunidad sistémica; no obstante, su absorción disminuye con el tiempo (cierre intestinal). Posteriormente, el animal recibe leche materna que empapa las paredes intestinales y proporciona inmunidad local a través de la IgA (Galitsopouloua, Michaelidoua, Menexesb \& Alichanidisa, 2015). Hasta los 28-30 días de edad, el lechón no es capaz de producir su propia actividad inmunológica de manera apropiada, por tanto, cualquier manifestación de estrés, bien sea digestivo, de manejo o combinado va a afectar al lechón desde el punto de vista inmunológico (Ciro, 2012). Debido a esto, los lechones presentan una baja respuesta inmunológica durante el período del posdestete.
Durante el período posdestete, la colonización bacteriana y la infección intestinal por enteropatógenos tienen un impacto en el número de linfocitos intraepiteliales asociados con el tracto intestinal (Parra, Agudelo, Sanín, Forero, Muskus \& López, 2013). Esto se debe principalmente a que el desarrollo de la inmunidad pasiva proveniente del calostro de la madre cae drásticamente a partir de la segunda semana de vida, y la inmunidad activa toma mayor fuerza a partir de la cuarta semana, presentando una baja respuesta inmunológica durante el destete (Gómez, Vergara \& Argote, 2008; Jurado, Ramírez \& Martínez, 2013). Además, durante este período el lechón suele presentar reacciones de hipersensibilidad a antígenos de los ingredientes vegetales en el alimento, especialmente las leguminosas, lo que aumenta la aparición de episodios diarreicos (Heo, Opapeju, Pluske, Kim, Hampson \& Nyachoti, 2013).

Los lechones se encuentran diariamente enfrentados a agentes patógenos presentes en las producciones; por esto surge la necesidad de buscar diferentes estrategias que permitan tener un mayor control sobre el 
estatus sanitario de los lechones. Un indicador del estado inmunitario de los animales es la necesidad del uso de la vacunación.

Mycoplasma hyopneumoniae es el principal agente etiológico de la neumonía enzoótica porcina, y es uno de los principales patógenos implicados en el complejo respiratorio porcino (Thacker \& Minion, 2012). La infección por $M$. hyopneumoniae se produce al nivel mundial, resultando en importantes pérdidas económicas en la industria porcina. Actualmente se dispone de vacunas inactivadas y vacunas vivas con adyuvante, que se utilizan comúnmente para controlar la enfermedad y las pérdidas asociadas con el rendimiento, y su eficacia se ha demostrado en diversos ensayos de campo y condiciones experimentales (Maes, 2014). Por otra parte, el Circovirus porcino tipo 2 (PCV2) es el agente causal del síndrome del desmedro multisistémico posdestete (PMWS), una enfermedad multifactorial que afecta a los cerdos de cría y de engorde (Segalés, 2012). Este síndrome se caracteriza por una pérdida progresiva de peso, y las infecciones oportunistas o secundarias son reportadas como comunes (Grau-Roma, Frailea \& Segalés, 2012). Todo el cuadro de la enfermedad es altamente sugestivo de una inmunodeficiencia adquirida.

Algunos alimentos utilizados para la nutrición de lechones durante la etapa posdestete sufren procesos de adición de antibióticos en niveles subterapéuticos, aportando propiedades que controlan o destruyen microorganismos presentes en el tracto gastrointestinal, lo que hace que los animales sean menos susceptibles a estímulos antigénicos y gracias a esto puedan actuar al mismo tiempo como promotores de crecimiento (Martin, Pieper, Kröger, Boroojenia, Vahjen, Neumann, Van Kessel, \& Zentek, 2012). Actualmente se han creado controversias acerca del uso de dichos antibióticos en la producción porcina intensiva, debido a que se han considerado como factores de riesgo de transmisión de resistencias microbianas a antibióticos utilizados en medicina humana (Claesson, Cusacka, O'Sullivan, Greene-Diniza, deWeerd, Flannery, et al., 2011; Allen, Levine, Looft, Bandrick \& Casey, 2013).
Como una alternativa al uso de antibióticos, se han utilizado microorganismos vivos que mejoran el equilibrio microbiano intestinal y que aportan beneficios a la salud del animal (Zacharof \& Lovitt, 2012). Estos microorganismos son denominados probióticos (Saad, Delattre, Urdaci, Schmitter, \& Bressollier, 2013). Debido a lo anterior, el objetivo de nuestro trabajo fue determinar el efecto de probióticos (L. acidophilus, L. casei y E. Faecium) sobre las poblaciones de células inmunes a nivel sanguíneo de cerdos en crecimiento.

\section{Materiales y métodos}

\section{Consideraciones éticas}

Todos los procedimientos experimentales fueron llevados a cabo de acuerdo con las guías propuestas por "The International Guiding Principles for Biomedical Research Involving Animals" (CIOMS, 1985). Esta investigación fue avalada por el Comité de Ética en la Experimentación Animal de la Universidad Nacional de Colombia, sede Medellín (CEMED012 del 14 de mayo de 2012).

\section{Localización}

El trabajo de campo se realizó bajo condiciones comerciales en la granja Caña Brava, ubicada en el municipio de Gómez Plata (Antioquia, Colombia), vereda "La Bonita", localizada a 1540 metros sobre el nivel del mar (msnm), con una temperatura promedio de $21^{\circ} \mathrm{C}$, perteneciente a la zona de vida de bosque húmedo premontano (bh-PM).

\section{Animales}

Se utilizaron 80 lechones (hembras y machos) del cruce duroc x pietran, buscando asemejar las condiciones normales de producción, destetados exactamente a los 21 días de edad, con un peso aproximado de $6 \pm 0.5$ $\mathrm{kg}$, los cuales fueron alojados en grupos de 8 animales durante el período de levante. Cada una de las instalaciones o corrales estaba provista de comederos de canoa y bebedero automático con agua a voluntad, y contaba con temperatura controlada $\left(26 \pm 3{ }^{\circ} \mathrm{C}\right)$. La 
dieta comercial ofrecida fue enriquecida con vitaminas, minerales y lisina HCL. Las dietas se balancearon para cumplir con todos los mínimos nutricionales requeridos y propuestos por el NRC (2012) (tabla 1). La cantidad de alimento ofrecido a los lechones por jaula fue administrada de acuerdo con la tabla dietaria que corresponde para la etapa productiva (levante). Asimismo, el agua de bebida que contenía las cepas probióticas se ofreció diariamente desde el día 1 del destete hasta finalizar el experimento, el cual tuvo una duración de 30 días. Durante la lactancia no se suministró alimento sólido a los lechones.

Tabla 1. Composición proximal

\begin{tabular}{l|c}
\hline Proteína cruda (\%) & 22.0 \\
\hline Extracto etéreo (\%) & 6.0 \\
\hline Cenizas (\%) & 8.0 \\
\hline Fibra (\%) & 5.0 \\
\hline Humedad (\%) & 13.0 \\
\hline
\end{tabular}

Fuente: elaborado por el autor

\section{Instalaciones y equipos}

Las instalaciones fueron desinfectadas y encaladas para la llegada de los lechones. Los cerdos fueron alojados en corrales con piso de cemento ( $1.5 \times 3$ metros). Del día 0 al 15 del experimento, los corrales estaban dotados de lechoneras (cuarto para el mantenimiento de la temperatura de los lechones) y cama de viruta de madera dentro de la lechonera. Para mantener la temperatura homogénea el corral estaba provisto de cortinas.

\section{Dietas}

Los animales fueron alimentados con dos dietas: dieta comercial con y sin la adición de antibiótico. Los diferentes probióticos (Lactobacillus casei, Lactobacillus acidophilus o Enterococcus faecium) se suministraron en el agua de bebida de los animales que consumieron la dieta comercial sin antibiótico, así:

- Dieta 1 Control (D1): Alimento comercial sin antibiótico, sin adición de cepa probiótica en el agua de bebida.

- Dieta 2 (D2): Alimento comercial con antibiótico, sin adición de cepa probiótica en el agua de bebida.
- Dieta 3 (D3: D1+ L. Acidophilus): Alimento comercial sin antibiótico, con adición de la cepa comercial probiótica L. Acidophilus en el agua de bebida.

- Dieta 4 (D4: D1+ L. Casei): Alimento comercial sin antibiótico, con adición de la cepa comercial probiótica $L$. Casei en el agua de bebida.

- Dieta 5 (D5: D1+ E. faecium): Alimento comercial sin antibiótico, con adición de la cepa comercial probiótica $E$. faecium en el agua de bebida.

La cantidad del probiótico adicionado (relación $\mathrm{v} / \mathrm{p}$ ) fue del $20 \%$ con respecto al peso de la ración utilizada, siguiendo las instrucciones para su preparación y adición, según lo recomendado por el fabricante. La inclusión de los probióticos en el agua de bebida se realizó (dos veces al día) por mezclado directo de un litro de agua con $30 \mathrm{gr}$ de azúcar comercial, la cual fue adicionada en un tanque de 20 L de agua, y evaluada por medio de análisis microbiológicos semanales para garantizar poblaciones mínimas de $10^{8} \mathrm{UFC} / \mathrm{mL}$ con una viabilidad adecuada. El alimento utilizado en el estudio estuvo libre de antibióticos (excepto la dieta D2), ya que no fue de interés modificar la dieta, sino la incorporación de los probióticos como una alternativa al uso de antibióticos. 
Las dietas experimentales se proporcionaron durante 30 días a partir del día del destete (día 21 de vida).

\section{Toma de muestras}

\section{Sangre para la determinación de células inmunes}

Se tomaron de la vena yugular $10 \mathrm{~mL}$ de sangre por animal en un tubo con anticoagulante los días 1, 15, y 30 posdestete. Las muestras se mantuvieron en frío para su transporte al laboratorio donde fueron analizadas.

\section{Sangre para la determinación de títulos de anticuerpos vacunales}

Se tomó una muestra de sangre $(5 \mathrm{~mL}$ aproximadamente) directamente de la vena yugular de cada animal, la cual fue almacenada en tubos de ensayos tapa roja debidamente identificados, e inmediatamente centrifugada para separar el suero. Las muestras fueron almacenadas a $-70{ }^{\circ} \mathrm{C}$ hasta la realización de los análisis de laboratorio (títulos de anticuerpos vacunales contra Micoplasma y Circovirus porcino tipo 2).

\section{Análisis de las muestras}

\section{Análisis de las poblaciones de células inmunes en sangre}

Luego de la toma de muestras, dichas muestras fueron fijadas en portaobjetos, los cuales fueron transportados al laboratorio para su posterior análisis. Se realizó un extendido de sangre periférica, luego el portaobjetos fue sometido a tinción con el colorante de Wright y finalmente se realizó el conteo diferencial de células para cada muestra (Yavari, Haghkhah, Ahmadi, Gheisari, \& Nazifi, 2009). Se realizó un conteo general para observar las poblaciones de eosinófilos, basófilos, neutrófilos, monocitos y linfocitos para determinar cuales se encontraban en mayor porcentaje. Se contaron cien a doscientos campos con ayuda del contador manual de células bajo el lente de 100X y se realizó el cálculo general del porcentaje de cada población de leucocitos (Ahmadi, Nazifi, \& Ghaisari, 2006)

\section{Análisis estadístico}

Se realizó un diseño de bloques al azar (dos bloques) en un arreglo de medidas repetidas en el tiempo (Steel \& Torrie, 1985) donde los animales fueron aleatorizados a uno de 5 tratamientos (alimento comercial sin probióticos, alimento sin antibióticos y sin probióticos, y alimento con adición de L. Casei, L. Acidophilus y E. Faecium). El análisis estadístico fue desarrollado usando el procedimiento GLM del (SAS $®, 2007)$. Las diferencias entre tratamientos y períodos fueron determinadas por LS means (media de mínimos cuadrados); además, se utilizó una prueba de Duncan para detectar significancia $(P<0.05)$ entre las medias.

\section{Resultados}

Los cerdos que consumieron las dietas experimentales no presentaron signo alguno de enfermedad que causara su retiro o sacrificio inmediato, es decir su rendimiento durante la fase de producción avanzó sin problemas fisiológicos, como decaimiento, alta temperatura corporal o bajo desarrollo, y bajo los parámetros esperados de rendimiento. Además, al nivel en que se fijó el suministro diario de alimento y agua no generó sobrantes.

En este experimento no se encontró interacción estadística entre las diferentes dietas experimentales dentro de cada período de evaluación para ninguna de las variables en estudio, por lo que no fue necesario analizar y desglosar dichos factores de manera independiente.

Los cambios de las poblaciones celulares inmunes sanguíneas estudiadas entre cada una de las dietas y períodos de exposición se pueden observar en la tabla 2. Con respecto a las poblaciones celulares inmunes sanguíneas, se presentó un aumento estadístico significativo $(P<0.01)$ entre las diferentes dietas evaluadas dentro de cada período de evaluación, donde D1 obtuvo valores menores respecto a D2 y frente a las dietas con probióticos, donde los animales en D5 reportaron los valores más altos en las poblaciones de células inmunes sanguíneas. Para las mismas variables en 
estudio, se presentó diferencia significativa estadística entre los diferentes días de muestreo dentro de cada una de las dietas
$(P<0.05)$, donde en el día 30 posdestete se presentaron los mayores valores para cada dieta en estudio.

Tabla 2. Caracterización de poblaciones de células inmunes sanguíneas en cerdos, con y sin adición de cepas probióticas durante $\mathbf{3 0}$ días posdestete

\begin{tabular}{|c|c|c|c|c|c|c|c|}
\hline VARIABLE & Día & D1 & D2 & D3 & D4 & D5 & EEM \\
\hline \multirow{3}{*}{$\begin{array}{c}\text { Recuento } \\
\text { Leucocitos } \\
\text { totales } \\
\text { (Cél. } X 10^{3} / \mu \mathrm{L} \text { ) }\end{array}$} & 1 & $12,04^{x}$ & $12,12^{x}$ & $12,09^{x}$ & $12,04^{x}$ & $12,12^{x}$ & \multirow{18}{*}{0,03} \\
\hline & 15 & $12,56^{A, Y}$ & $13,73^{\mathrm{B}, Y}$ & $13,95^{\mathrm{C}, \mathrm{Y}}$ & $15,47^{\mathrm{D}, \mathrm{Y}}$ & $15,97^{\mathrm{E}, \mathrm{Y}}$ & \\
\hline & 30 & $13,67^{A, Z}$ & $14,95^{\mathrm{B}, \mathrm{Z}}$ & $15,13^{\mathrm{c}, \mathrm{z}}$ & $15,96^{\mathrm{D}, \mathrm{z}}$ & $16,44^{\mathrm{E}, \mathrm{Z}}$ & \\
\hline \multirow{3}{*}{ Linfocitos (\%) } & 1 & $40,51^{x}$ & $40,61^{x}$ & $40,52^{x}$ & $40,57^{x}$ & $40,58^{x}$ & \\
\hline & 15 & $42,72^{\mathrm{A}, \mathrm{Y}}$ & $43,41^{B, Y}$ & $44,15^{\mathrm{C}, \mathrm{Y}}$ & $47,52^{\mathrm{D}, \mathrm{Y}}$ & $49,48^{\mathrm{E}, Y}$ & \\
\hline & 30 & $44,62^{A, Z}$ & $47,52^{\mathrm{B}, \mathrm{Z}}$ & $49,48^{c, z}$ & $49,52^{c, z}$ & $51,62^{\mathrm{D}, \mathrm{Y}}$ & \\
\hline \multirow{3}{*}{ Monocitos (\%) } & 1 & $2,15^{x}$ & $2,14^{x}$ & $2,15^{x}$ & $2,14^{x}$ & $2,15^{x}$ & \\
\hline & 15 & $2,21^{\mathrm{A}, \mathrm{Y}}$ & $2,38^{\mathrm{B}, \mathrm{Y}}$ & $2,55^{\mathrm{C}, \mathrm{Y}}$ & $2,84^{\mathrm{D}, \mathrm{Y}}$ & $3,08^{E, Y}$ & \\
\hline & 30 & $2,31^{A, Z}$ & $2,54^{\mathrm{B}, \mathrm{Z}}$ & $2,82^{\mathrm{c}, \mathrm{z}}$ & $3,05^{\mathrm{D}, \mathrm{Z}}$ & $3,25^{\mathrm{E}, \mathrm{Z}}$ & \\
\hline \multirow{3}{*}{ Neutrófilos (\%) } & 1 & $31,34^{x}$ & $31,39^{x}$ & $31,29^{x}$ & $31,31^{x}$ & $31,24^{x}$ & \\
\hline & 15 & $33,38^{A, Y}$ & $34,23^{\mathrm{B}, Y}$ & $34,34^{\mathrm{B}, \mathrm{Y}}$ & $35,74^{\mathrm{C}, \mathrm{Y}}$ & $37,08^{\mathrm{D}, \mathrm{Y}}$ & \\
\hline & 30 & $34,02^{A, Z}$ & $35,74^{\mathrm{B}, \mathrm{Z}}$ & $36,89^{c, z}$ & $37,02^{\mathrm{D}, \mathrm{Z}}$ & $39,21^{\mathrm{E}, \mathrm{Z}}$ & \\
\hline \multirow{3}{*}{ Eosinófilos (\%) } & 1 & $0,41^{x}$ & $0,48^{x}$ & $0,49^{x}$ & $0,42^{x}$ & $0,42^{x}$ & \\
\hline & 15 & $0,59^{A, Y}$ & $0,78^{\mathrm{B}, \mathrm{Y}}$ & $0,97^{\mathrm{C}, Y}$ & $0,107^{D, Y}$ & $0,126^{E, Y}$ & \\
\hline & 30 & $0,81^{A, Z}$ & $0,99^{\mathrm{B}, \mathrm{z}}$ & $1,12^{\mathrm{c}, \mathrm{z}}$ & $1,29^{\mathrm{D}, \mathrm{z}}$ & $1,51^{\mathrm{E}, \mathrm{Z}}$ & \\
\hline \multirow{3}{*}{ Basófilos (\%) } & 1 & 0,11 & 0,12 & 0,1 & 0,11 & 0,12 & \\
\hline & 15 & 0 & 0 & 0 & 0,095 & 0,04 & \\
\hline & 30 & 0 & 0 & 0 & 0 & 0 & \\
\hline
\end{tabular}

D1: Alimento comercial sin antibiótico y sin probiótico; D2: Alimento comercial + antibiótico; D3: Alimento comercial sin antibiótico + L. acidophilus; D4: Alimento comercial sin antibiótico + L. casei; D5: Alimento comercial sin antibiótico + E. faecium. A,B,C,D,E Dentro de una misma fila, medias con diferente superíndice son estadísticamente diferentes $(P<0.05)$. ${ }^{X, Y, z}$ Dentro de una misma columna, medias con un superíndice común (por variable en estudio) no difieren estadísticamente $(\mathrm{P}<0.05)$. EEM: Error estándar de la media.

Las figuras 1 a 6 ilustran el comportamiento de las poblaciones de células inmunes sanguíneas, de acuerdo con las diferentes dietas experimentales, durante el tiempo del experimento.

Con respecto a la producción de anticuerpos sanguíneos contra Micoplasma y Circovirus (tabla 3), se presentó una disminución estadística significativa $(P<0.01)$ entre las diferentes dietas evaluadas dentro de cada período de evaluación, donde D1 obtuvo valores menores respecto a D2 y frente a dietas con probióticos; además, los animales en D5 reportaron los valores más altos en la producción de anticuerpos sanguíneos contra Micoplasma. De igual manera, la producción de anticuerpos contra Circovirus porcino (tabla 3) presentó un aumento estadístico significativo $(P<0.01)$ entre las diferentes dietas, donde D5 presentó los mayores valores en comparación con las otras dietas suplementadas con probióticos (D3 y D4) y, especialmente, con la dieta adicionada con antibiótico. Para las 
mismas variables en estudio, se presentó diferencia significativa estadística entre los diferentes días de muestreo dentro de cada una de las dietas $(\mathrm{P}<0.05)$, donde en el día 30 posdestete se presentaron los mayores valores para cada dieta en estudio.

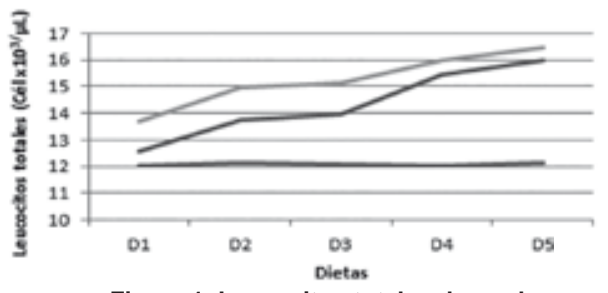

Figura 1. Leucocitos totales de cerdos, con y sin adición de cepas probióticas durante 30 días posdestete

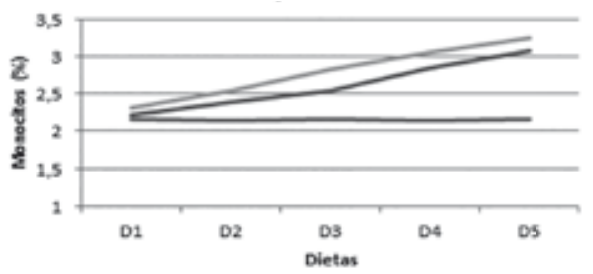

Figura 3. Monocitos de cerdos, con y sin adición de cepas probióticas durante $\mathbf{3 0}$ días posdestete

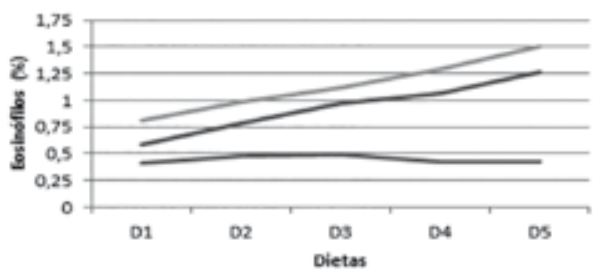

Figura 5. Eosinófilos de cerdos, con y sin adición de cepas probióticas durante 30 días posdestete
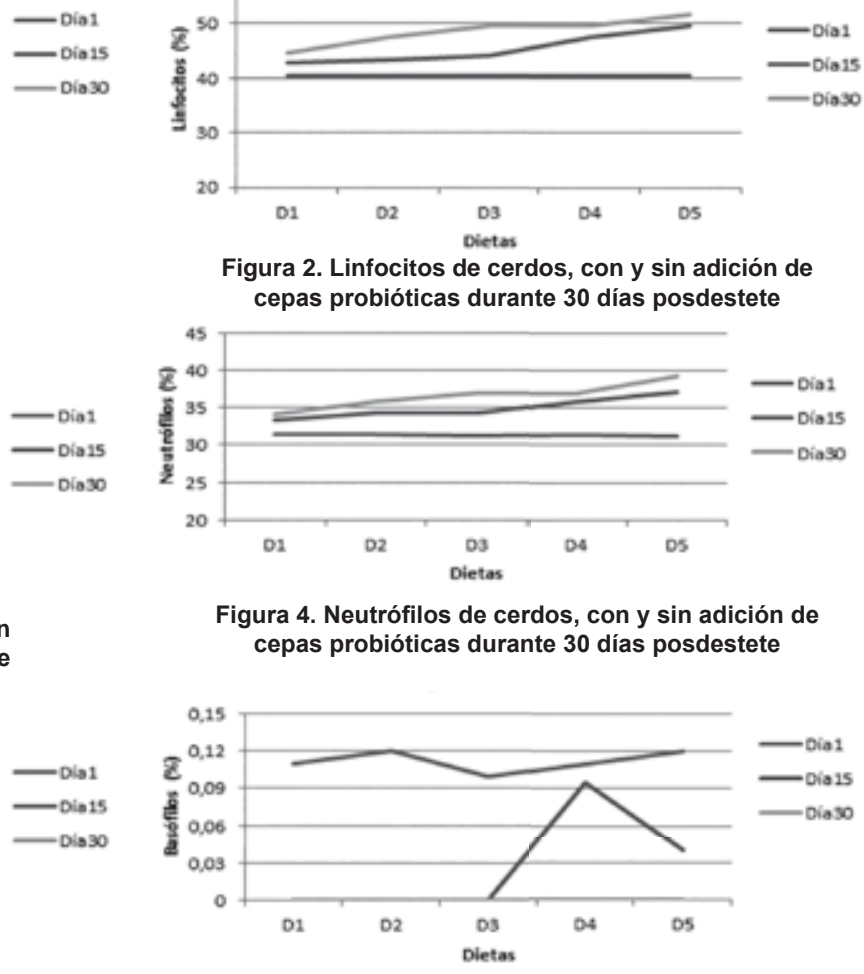

Figura 6. Basófilos de cerdos, con y sin adición de cepas probióticas durante $\mathbf{3 0}$ días posdestete

Tabla 3. Producción de anticuerpos vacunales en cerdos con y sin adición de cepas probióticas durante 30 días posdestete

\begin{tabular}{c|c|c|c|c|c|c|c}
\hline VARIABLE & Día & D1 & D2 & D3 & D4 & D5 & \multirow{2}{*}{ EEM } \\
\hline \multirow{4}{*}{ Micoplasma } & 1 & ND & ND & ND & ND & ND & \\
\cline { 2 - 6 } & 15 & $0,41^{\mathrm{A}, \mathrm{X}}$ & $0,45^{\mathrm{A}, \mathrm{X}}$ & $0,59^{\mathrm{B}, \mathrm{X}}$ & $0,65^{\mathrm{C}, \mathrm{X}}$ & $0,75^{\mathrm{D}, \mathrm{X}}$ & \multirow{2}{*}{0,01} \\
\cline { 2 - 6 } & $\mathbf{3 0}$ & $0,26^{\mathrm{A}, \mathrm{Y}}$ & $0,25^{\mathrm{A}, \mathrm{Y}}$ & $0,27^{\mathrm{A}, \mathrm{Y}}$ & $0,32^{\mathrm{B}, \mathrm{Y}}$ & $0,41^{\mathrm{C}, \mathrm{Y}}$ & \\
\hline \multirow{3}{*}{ Circovirus } & 1 & $\mathrm{ND}$ & $\mathrm{ND}$ & $\mathrm{ND}$ & $\mathrm{ND}$ & $\mathrm{ND}$ & \\
\cline { 2 - 7 } & 15 & $28,4^{\mathrm{A}, \mathrm{X}}$ & $30,7^{\mathrm{B}, \mathrm{X}}$ & $35,2^{\mathrm{C}, \mathrm{X}}$ & $47,5^{\mathrm{D}, \mathrm{X}}$ & $55,1^{\mathrm{E}, \mathrm{X}}$ & \\
\cline { 2 - 7 } & 30 & $20,6^{\mathrm{A}, \mathrm{Y}}$ & $25,7^{\mathrm{B}, \mathrm{Y}}$ & $27,4^{\mathrm{C}, \mathrm{Y}}$ & $35,5^{\mathrm{C}, \mathrm{Y}}$ & $46,6^{\mathrm{D}, \mathrm{Y}}$ & \\
\hline
\end{tabular}

D1: Alimento comercial sin antibiótico y sin probiótico; D2: Alimento comercial+ antibiótico; D3: Alimento comercial sin antibiótico+ L. acidophilus; D4: Alimento comercial sin antibiótico+ L. casei; D5: Alimento comercial sin antibiótico+ E. faecium. ${ }^{A, B, C, D, E}$ Dentro de una misma fila, medias con diferente superíndice son estadísticamente diferentes $(P<0.05) .{ }^{X}, \mathrm{Y}, \mathrm{Z}$ Dentro de una misma columna, medias con un superíndice común (por variable en estudio) no difieren estadísticamente $(\mathrm{P}<0.05)$. EEM: Error estándar de la media. 
Las figuras 7 y 8 ilustran el cambio en la producción de anticuerpos contra Micoplasma y Circovirus porcino (respectivamente), de acuerdo con las diferentes dietas experimentales, durante el tiempo del experimento.

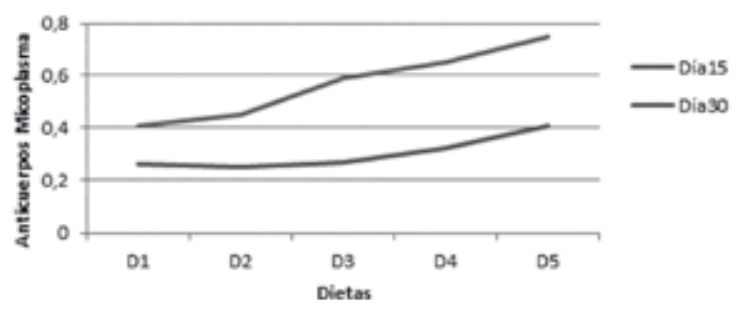

Figura 7. Anticuerpos Micoplasma de cerdos, con y sin adición de cepas probióticas durante 30 días posdestete

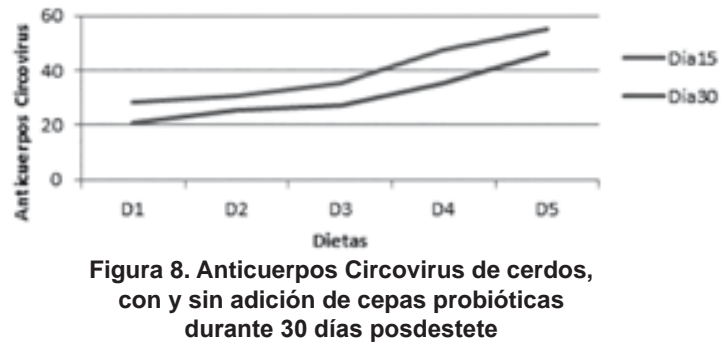

\section{Discusión}

El período posdestete se caracteriza por una reducción inmediata, pero transitoria, en el consumo de alimento que conduce a un estado de desnutrición y de retraso en el crecimiento; y por la aparición de signos de inflamación intestinal temprana, como signo del desequilibrio bacteriano y la presencia de antígenos alimentarios de la nueva dieta, que llevan a la activación descontrolada del sistema inmune intestinal y sistémico (Ciro, 2012). Después del destete, pueden ser detectadas respuestas inmunes a ingredientes del alimento, a raíz de la cual, el animal va adquiriendo tolerancia al alimento, aunque esto pueda tomar algún tiempo (Pasternak, Hon \& Wilson, 2014).

Se ha demostrado que las sustancias que mejoran o estimulan la respuesta inmune (sustancias adyuvantes o inmunomoduladores), para nuestro caso los probióticos, pueden mejorar los mecanismos de defensa inespecíficos del huésped y activar las células implicadas en la respuesta inmune innata y/o adaptativa (BatistaDuharte Lindbladb \& Oviedo-Ortac, 2011). Los probióticos pueden activar el sistema inmunitario mediante la estimulación de uno, o todos los tipos de respuestas, y tienen el potencial para ejercer un efecto mediante la prevención de enfermedades en las que el sistema inmune desempeña un papel fundamental. Esto, como se observó en los resultados de nuestra investigación, está dado por el aumento en la población de células inmunes sanguíneas preparando al hospedero para un desafío al cambio de ambiente y de alimento.

La modulación de la respuesta inmune sistémica y secretora está bien establecida en ratones y otros animales de experimentación (cerdos), donde se ha demostrado la proliferación de los órganos del sistema inmune (Placas de Peyer); la estimulación de fagocitos y células Natural Killer (NK); el incremento en la liberación de citoquinas (TNF-alfa, INFgamma) y defensinas; y el aumento en la producción de anticuerpos específicos durante la administración de virus, toxinas y bacterias (Zhu, Gao, Wu \& Qin, 2013). Algunos estudios 
demostraron que tanto los microorganismos probióticos, como los componentes de su pared celular (peptidoglicanos, lipopolisacáridos), el ADN, y los metabolitos tienen propiedades inmunomodulatorias al nivel intestinal (Roberfroid, Gibson, Hoyles, McCartney, Rastall, Rowland, et al., 2010). De acuerdo con nuestra investigación, podríamos afirmar que los lechones suplementados con las cepas probióticas mostraron una rápida adaptación a los desafíos del destete. Esto exhibido por el aumento significativo de las poblaciones celulares inmunes sanguíneas (linfocitos, monocitos y granulocitos) en los lechones suplementados con cepas probióticas. Los lechones suplementados con el probiótico E. faecium mostraron de manera significativa un mayor efecto en el nivel inmune, en comparación con los demás grupos suplementados, contrario a lo sucedido con los lechones de los grupos control (antibiótico y sin adición de cepa probiótica ni antibiótico), que exhibieron menores niveles de células inmunes sanguíneas.

El papel fundamental de la microbiota intestinal en el desarrollo y la maduración de la respuesta inmune se ha demostrado claramente evidenciando problemas significativos en el desarrollo y la maduración de la respuesta inmune, tanto de tipo celular como humoral, en modelos animales en ausencia de la microbiota intestinal (Di Mauro, Neu, Riezzo, Raimondi, Martinelli, Francavilla, et al., 2013). Los probióticos mejoran la inmunidad a través de la inmunomodulación donde interactúan con células presentadoras de antígeno, es decir, los macrófagos y las células dendríticas. Tras la interacción con probióticos estas células secretan ciertas citoquinas que regulan la función de las células $T$ reguladoras, lo que resulta en la inmunomodulación (Forsythe \& Bienenstock, 2010). Esto da como resultado un sistema inmunológico eficaz disminuyendo la susceptibilidad a diversas inflamaciones y alergias (tolerancia) a través de diversos ejes tracto grastrointestinal (TGI) -órganos de inmunomodulación, es decir, eje TGI-cerebro, TGI-, pulmón y TGI-piel (Forsythe, 2011; Philpott, Gibson \& Thien, 2011; Bercik, Collins \& Verdu, 2012). Dicha inmunomodulación ocurre simultáneamente de dos maneras: i) por la presencia de antígenos alimentarios de la nueva dieta y ii) por el consumo de bacterias probióticas que ayudan al equilibrio bacteriano y a la activación controlada del sistema inmune. Por lo anterior, se ha sugerido que la selección de una cepa probiótica apropiada para su inclusión en una preparación probiótica debe hacerse sobre la base de su capacidad para inducir una respuesta inmune mejorada (Galdeano, Dogi, Bonet, de Moreno de LeBlanc \& Perdigón, 2013). De acuerdo con lo anterior y con los resultados obtenidos en nuestra investigación, se confirma que las bacterias utilizadas, especialmente E. faecium, cumplen con el criterio de inmunomodulación para la selección como cepa probiótica, ya que ayudan a la presentación de respuestas inmunes controladas en situaciones de alto estrés, como el destete.

Dong et al. (2010) demostraron que el Lactobacillus casei indujo la activación preferencial de las células citotóxicas. La maduración de células NK y células T (CD8+) por los macrófagos contribuye a una respuesta basada en linfocitos $T$ ayudadores (Critchfield, van Hemert, Ash, Mulder, \& Ashwood, 2011). En un estudio in vitro, se demostró que luego de ser fagocitada la cepa probiótica $L$. casei por los monocitos, se estimuló directamente la secreción no solo de citoquinas proinflamatorias, como IL-12 y TNF- $\alpha$, sino también citoquinas antinflamatorias, IL-10 (Dong, Rowland \& Yaqood, 2012). Por consiguiente, se propone que los monocitos desempeñan un papel importante en la inmunomodulación inducida por probióticos.

El efecto de mejora de la inmunidad por administración de bacterias acidolácticas (BAL) también se demostró en un modelo de inmunosupresión en ratones inducida por corticoides o la administración de ampicilina, mostrando un aumento en el número de leucocitos y una mejora en la actividad fagocítica de los macrófagos peritoneales (Galdeano, Dogi, Bonet, de Moreno de LeBlanc \& Perdigón, 2013).

Varios estudios en humanos han demostrado que los probióticos mejoran la actividad de las células NK (Olivares, Díaz-Ropero, Sierra, Lara-Villoslada, Fonollá, Navas, 2007; Takeda \& Okumura, 2007; Zanini, Marzotto, 
Castellazzi, Borsari, Dellaglio, \& Torriani, 2007, Biagi, Candela, Fairweather-Tait, Franceschi \& Brigidi, 2012). Los resultados presentados por Dong et al. (2010) mostraron una mejora directa de la actividad de células NK en células mononucleares de sangre periférica humana (CMSP) estimuladas por Lactobacillus casei in vitro, lo que está de acuerdo con otro estudio in vitro (Dong et al., 2012) empleando dicha cepa muerta por calor y que muestra que la activación y la actividad de las células NK fueron mejoradas por estimulación del Lactobacillus casei. Estos datos sugieren que las cepas probióticas podrían potenciar la destrucción de las células infectadas en el cuerpo y mejorar la defensa del huésped.

Los estudios in vitro y en modelos animales han indicado que los organismos probióticos pueden mejorar tanto la respuesta inmune específica, como también la respuesta inmune no específica mediante la activación de los macrófagos, la alteración de la expresión de citoquinas, aumento de la actividad de las células NK, y aumento del nivel de inmunoglobulina (van Baarlen, Troost, Hemert Svan, van der, de Groota, Hooivelda, et al., 2009). Estas observaciones, junto con los resultados obtenidos en nuestro estudio, indican que los monocitos son importantes para los efectos inmunomodulatorios en el huésped.

Referente a la producción de anticuerpos, los ensayos de campo mostraron que las vacunas de $M$. hyopneumoniae tuvieron efectos beneficiosos en varias camadas clínicamente afectadas por la neumonía enzoótica. Además de ser eficaz y segura, la vacuna también debe ser rentable (Siugzdaite, Garlaite \& Urbsiene, 2003). Por su parte, en condiciones de campo, todas las vacunas comerciales para Circovirus porcino (PCV2) han sido capaces de reducir el impacto del Síndrome del desmedro multisistémico posdestete (PMWS) disminuyendo significativamente la mortalidad en los lechones (Kekarainen, McCullough, Fort, Fossum, Segalés, \& Allan, 2010), además de disminuir la frecuencia de co-infecciones en cerdos vacunados en comparación con cerdos no vacunados (Desrosiers, Clark, Tremblay, Tremblay \& Polson, 2009; Segalés, 2012). Sin embargo, la vacunación tiene sus limitaciones debido a que la protección es incompleta, la eficacia varía de una camada a otra, y a que en última instancia, no evitan la adherencia/ colonización de patógenos del hospedero.

Los títulos de anticuerpos vacunales contra Micoplasma y Circovirus porcino presentaron diferencias estadísticas significativas de acuerdo con la cepa probiótica suplementada. Los lechones suplementados con E. faecium presentaron una mayor producción de anticuerpos contra Micoplasma y Circovirus porcino, en comparación con los demás grupos suplementados, la dieta comercial y el grupo control. Los probióticos en el organismo de un animal sano pueden estimular la respuesta inmune no específica y optimizar la protección inmune (Musa, Wu, Zhu, Seri \& Zhu, 2009).

\section{Conclusión}

El destete está asociado con múltiples factores que generan la presentación de estrés en los animales, los cuales provocan un desequilibrio bacteriano, lo que puede contribuir a numerosas patogénesis de condiciones inflamatorias. La cepa de probiótico $E$. faecium pudo ayudar a mantener la funcionalidad sistémica y la respuesta inmune protectora de manera apropiada y controlada. La inclusión de probióticos, especialmente $E$. faecium, parece ser un elemento importante en el control de Micoplasma y Circovirus en la producción porcina, reflejado en el aumento en la producción de anticuerpos que favorecen la salud del animal.

\section{Referencias bibliográficas}

- $\quad$ Ahmadi, M.; Nazifi, S. \& Ghaisari, H. (2006). Comparison of hormonal changes of estrus cycle with cytology of cervical mucosa \& hematological parameters in dairy heifers. Comp. Clin. Pathol. 15, 94-97.

- Allen, H.; Levine U.; Looft T.; Bandrick M. \& Casey, T. (2013). Treatment, promotion, commotion: antibiotic alternatives in foodproducing animals. Trends in Microbiology, 21(3).

- Batista-Duhartea, A.; Lindbladb, E. \& OviedoOrtac E. (2011). Progress in understanding 
adjuvant immunotoxicity mechanisms. Toxicology Letters, 203(2), 97-105.

- $\quad$ Bercik, P.; Collins, S. \& Verdu, E. (2012). Microbes and the gut-brain axis. Neurogastroenterol Motil. 24(5), 405-413.

- Biagi, E.; Candela, M.; Fairweather-Tait, S.; Franceschi, C. \& Brigidi, P. (2012). Ageing of the human metaorganism: the microbial counterpart. Age (Dordr), 34(1), 247-267.

- Council for International Organizations of Medical Sciences. (1985). International Guiding Principles for Biomedical Research Involving Animals, Geneva: CIOMS.

- Ciro, J. (2012). Efecto de la inflamación en yeyuno inducida por lipopolisacáridos (LPS) de E. coli sobre la expresión de proteínas de arquitectura y defensa en lechones destetos. [Tesis de Maestría en Ciencias Agrarias]. Medellín, Antioquia: Universidad Nacional de Colombia. Departamento de Producción Animal.

- Claesson, M.; Cusacka, S.; O’Sullivan, O.; Greene-Diniza, R.; De Weerd, H.; Flannery, E.; Marchesib, J.R.; Falushg, D.; Dinanb, T.; Fitzgeralda, G.; Stantonb, C.; Van Sinderena, D.; O'Connori, M.; Harnedyi, N.; O'Connori, K.; Henry, C.; O’Mahony, D.; Fitzgeralde, A.; Shanahan, F.; Twomey, C.; Hilla, C.; Rossb, R. \& O'Toolea, P. (2011). Composition, variability, and temporal stability of the intestinal microbiota of the elderly. Proc. Natl. Acad. Sci. 108(Suppl.1), 4586-4591.

- $\quad$ Critchfield, J.; Van Hemert, S.; Ash, M.; Mulder, L. \& Ashwood, P. (2011). Review Article The Potential Role of Probiotics in the Management of Childhood Autism Spectrum Disorders. Gastroenterology Research and Practice, 1-8.

- Desrosiers, R.; Clark, E.; Tremblay, D.; Tremblay, R. \& Polson, D. (2009). Use of a one-dose subunit vaccine to prevent losses associated with porcine Circovirus type 2. J Swine Health Prod. 17:148-54.

- $\quad$ Di Mauro, A.; Neu, J.; Riezzo, G.; Raimondi F.; Martinelli, D.; Francavilla, R. \& Indrio, F. (2013). Gastrointestinal function development and microbiota. BioMed Central. Italian Journal of Pediatrics, 39, 15.

- Dong, H.; Rowland, I.; Tuohy, K.; Thomas, L. \& Yaqoob, P. (2010). Selective effects of Lactobacillus casei Shirota on T cell activation, natural killer cell activity and cytokine production. Clinical and Experimental Immunology, 161, 378-388.
- Dong, H.; Rowland, I. \& Yaqoob, P. (2012). Comparative effects of six probiotic strains on immune function in vitro. British Journal of Nutrition, 108, 459-470.

- Forsythe, P. (2011). Probiotics and lung diseases. Chest, 139(4), 901-908.

- Forsythe, P. \& Bienenstock, J. (2010). Immunomodulation by commensal and probiotic bacteria. Immunol Invest. 39(4-5), 429-448.

- Galdeano, C.; Dogi, C.; Bonet, B.; De Moreno, A. \& Perdigón G. (2013). Probiotic Bacteria as Mucosal Immune System Adjuvants. En Bioactive Food as Dietary Interventions for Liver and Gastrointestinal Disease (Eds. Watson, R.). Burlington: Elsevier Science.

- $\quad$ Galitsopouloua, A.; Michaelidoua, A.; Menexesb G. \& Alichanidisa, E. (2015). Polyamine profile in ovine and caprine colostrum and milk. Food Chemistry, 173, 80-85.

- Gómez, I.; Vergara, D. \& Argote, F. (2008). Efecto de la dieta y edad del destete sobre la fisiología digestiva del lechón. Revista Biotecnología en el Sector Agropecuario y Agroindustrial, 6, 32-41.

- Grau-Romaa L.; Frailea, L. \& Segalés, J. (2011). Recent advances in the epidemiology, diagnosis and control of diseases caused by porcine Circovirus type 2 . The Veterinary Journal, 187(1), 23-32.

- Heo, J.; Opapeju, F.; Pluske, J.; Kim, J.; Hampson, D. \& Nyachoti, C. (2013). Gastrointestinal health and function in weaned pigs: a review of feeding strategies to control post-weaning diarrhoea without using in-feed antimicrobial compounds. J Anim Physiol Anim Nutr. 97, 207-237.

- Jurado, G.; Ramírez, T. \& Martínez, B. (2013). Evaluación in vivo de Lactobacillus plantarum como alternativa al uso de antibióticos en lechones. Revista MVZ Córdoba, 18(3), 36483657.

- Kekarainen, T.; McCullough, K.; Fort, M.; Fossum, C.; Segalés, J. \& Allan, G. (2010). Immune responses and vaccine-induced immunity against Porcine Circovirus type 2. Vet Immunol Immunopathol, 136(3-4), 185-93.

- Maes, D. (2014) Vaccination against Mycoplasma hyopneumoniae infection in pigs: Room for improvement. Guest Editorial. The Veterinary Journal, 200, 214-215

- Martin, L.; Pieper, R.; Kröger, S.; Boroojenia, F. G.; Vahjen, W.; Neumann, K.; Van Kessel, 
A. G. \& Zentek, J. (2012). Influence of age and Enterococcus faecium NCIMB 10415 on development of small intestinal digestive physiology in piglets. Animal Feed Science and Technology. 175, 65-75.

- $\quad$ Musa, H.; Wu, S.; Zhu, C.; Seri, H. \& Zhu, G. (2009). The Potential Benefits of Probiotics in Animal Production and Health. Journal of Animal and Veterinary Advances, 8(2), 313-321.

- Nutrient Requirements Council. (2012). The Nutrient Requirements of Swine. Eleventh Revised Edition. Washington, D.C. National Academy of Sciences.

- $\quad$ Olivares, M.; Díaz-Ropero, M.; Sierra, S.; LaraVilloslada, F.; Fonollá, J.; Navas, M.; Rodríguez, J. M. \& Xaus, J. (2007). Oral intake of Lactobacillus fermentum CECT5716 enhances the effects of influenza vaccination. Nutrition, 23, 254-60.

- $\quad$ Parra, S.; Agudelo, J.; Sanín, P.; Forero, D.; Muskus, L. \& López, H. (2013). Intestinal expression of pro-infiammatory cytokines induced by oral intake of lipopolysaccharide (LPS) from E. coli in weaned pigs. Rev Colom Cienc Pecua. 26(2).

- $\quad$ Pasternak, J.; Hon, Ng. S. \& Wilson, H. (2014). A single, low dose oral antigen exposure in newborn piglets primes mucosal immunity if administered with $\mathrm{CpG}$ oligodeoxynucleotides and polyphosphazene adjuvants. Veterinary Immunology and Immunopathology. 161, 3-4, 15, 211-221.

- $\quad$ Philpott, H.; Gibson, P. \& Thien, F. (2011) Irritable bowel syndrome - An inflammatory disease involving mast cells. Asia Pac Allergy. 1(1), 36-42.

- Roberfroid, M.; Gibson, G.; Hoyles, L.; McCartney, A.; Rastall, R.; Rowland, I.; Wolvers, D.; Watzl, B.; Szajewska, H.; Stahl, B.; Guarner, F.; Respondek, F.; Whelan, K.; Coxam, V.; Davicco, M. J.; Léotoing, L.; Wittrant, Y.; Delzenne, N. M.; Cani, P. D.; Neyrinck, A. \& Meheust, A. (2010). Prebiotic effects: metabolic and health benefits. Br J Nutr. 104(2), S1-63.

- $\quad$ Saad, N.; Delattre, C.; Urdaci, M.; Schmitter, J. \& Bressollier, P. (2013). An overview of the last advances in probiotic and prebiotic field. Food Science and Technology, 50, 1-16.
- Institute Inc. Statistical Analysis Systems Institute. (2007). SAS/STAT User's Guide, Version 9.1th Ed. Cary, NC: SAS Institute Inc.

- Segalés, J. (2012). Porcine Circovirus type 2 (PCV2) infections: Clinical signs, pathology and laboratory diagnosis. Virus Research, 164, 1-2, 10-19.

- Siugzdaite, J.; Garlaite, K. \& Urbsiene, D. (2003). Evaluation of antibody formation, daily weight gain and meat quality after vaccination of piglets against mycoplasma hyopneumoniae. Acta Veterinaria Hungarica, 51 (3), 273-281.

- Steel, R. \& Torrie, J. (1985). Principles and Procedures of Statistics: a biometrical approach (2a Ed), New York (USA): McGraw-Hill Book Co.

- $\quad$ Takeda, K. \& Okumura, K. (2007). Effects of a fermented milk drink containing Lactobacillus casei strain shirota on the human NK-cell activity. J Nutr. 137, 791S-3S.

- $\quad$ Thacker, E. \& Minion, F. (2012). Mycoplasmosis. In: Zimmerman, J. (Ed.). Diseases of Swine. lowa, USA: lowa State University Press, Ames.

- Van Baarlen, P.; Troost, F.; Hemert Svan, M.; Van der, de Vos, W.; De Groota, P.; Hooivelda, G.; Brummera, R. \& Kleerebezema, M. (2009). Differential NF-kB pathways induction by Lactobacillus plantarum in the duodenum of healthy humans correlating with immune tolerance. Proc Natl Acad Sci, 106, 2371-2376.

- Yavari, M.; Haghkhah, M.; Ahmadi, M.; Gheisari, H. \& Nazifi, S. (2009). Comparison of cervical and uterine cytology between defferent classification of postpartum endometritis and bacterial isolates in Holstein Dairy Cows. International Journal of dairy science, 4, 19-26.

- Zacharofa M. \& Lovittb R. (2012). Bacteriocins Produced by Lactic Acid Bacteria a Review Article. APCBEE Procedia, 2, 50-56.

- Zanini, K.; Marzotto, M.; Castellazzi, A.; Borsari, A.; Dellaglio, F. \& Torriani, S. (2007). The effects of fermented milks with simple and complex probiotic mixtures on the intestinal microblota and immune response of healthy adults and children. Int Dairy J. 17, 1332-43.

- Zhu Q.; Gao R.; Wu, W. \& Qin H. (2013). The role of gut microbiota in the pathogenesis of colorectal cancer. Tumor Biology, 34(3), 12851300. 